\title{
Féeries
}

Études sur le conte merveilleuX, XVII ${ }^{e}$ XIXe siècle

14 | 2017

Conte merveilleux et poésie

\section{Antoine Galland, Les Mille et une nuit. Contes arabes}

édition critique par Manuel Couvreur, avec la collaboration de Xavier Luffin, Paris, Honoré Champion, 2016, 2 vol., 1806 p.

Jean-Paul Sermain

\section{OpenEdition}

1 Journals

Édition électronique

URL : http://journals.openedition.org/feeries/1176

DOI : $10.4000 /$ feeries. 1176

ISSN : 1957-7753

Éditeur

UGA Éditions/Université Grenoble Alpes

Édition imprimée

ISBN : 978-2-37747-012-9

ISSN : $1766-2842$

Référence électronique

Jean-Paul Sermain, «Antoine Galland, Les Mille et une nuit. Contes arabes », Féeries [En ligne], 14 | 2017, mis en ligne le 31 juillet 2017, consulté le 24 septembre 2020. URL : http://journals.openedition.org/ feeries/1176; DOI : https://doi.org/10.4000/feeries.1176

Ce document a été généré automatiquement le 24 septembre 2020.

(c) Féeries 


\section{Antoine Galland, Les Mille et une nuit. Contes arabes}

édition critique par Manuel Couvreur, avec la collaboration de Xavier Luffin, Paris, Honoré Champion, 2016, 2 vol., 1806 p.

Jean-Paul Sermain

\section{RÉFÉRENCE}

Antoine Galland, Les Mille et une nuit. Contes arabes, édition critique par Manuel Couvreur, avec la collaboration de Xavier Luffin, Paris, Honoré Champion, 2016, 2 vol., $1806 \mathrm{p}$.

1 Ce $119^{e}$ volume de "Sources classiques", collection dirigée par Philippe Sellier et Dominique Descotes, est aussi le $6^{\mathrm{e}}$ de la «Bibliothèque des Génies et des Fées » dirigée par Nadine Jasmin.

2 Dans cette "bibliothèque ", ce texte, avec celui de Perrault, est le seul qui soit universellement connu et édité. Mais à l'inverse de Perrault, le texte n'avait bénéficié d'aucune édition critique. C'est donc une première, et elle convainc. L'établissement du texte est aisé parce qu'il n'existe qu'une édition du vivant de Galland (en dehors d'un retirage du tome II en 1705 qui a été soigneusement collationné, mais dont rien n'indique que Galland y a eu une part). Manuel Couvreur a donc surtout été attentif à restituer au mieux le texte original, il reproduit heureusement dans le texte et dans la table des matières le découpage en douze volumes, comme les découpages en nuits, en plus des titres des « histoires ». Il adopte les compromis de la collection et des éditions Champion: l'orthographe est modernisée mais beaucoup d'accords archaïques sont maintenus (mais pas ce qui concerne les modes et l'accord du participe passé). Par contre la ponctuation est pour l'essentiel conservée : on a donc accès à cet ancien système que les étudiants d'aujourd'hui adoptent spontanément en le mêlant au système moderne sans connaître ni l'un ni l'autre, les éditions archaïsantes n'étant pas sans danger sur des lecteurs si peu préparés. Les notes en nombre assez réduit s'en 
tiennent à trois types de remarques: les unes portent sur les écarts orthographiques par rapport à nos normes, les autres portent sur le vocabulaire, les troisièmes sur les réalités orientales. Les deux dernières visent à aider le lecteur, les premières s'adressent à des spécialistes de la langue.

3 L'ouvrage comporte plusieurs compléments fort utiles, outre la bibliographie et le résumé des histoires, la liste des "variantes" de l'éditeur et de Galland, un très précieux glossaire qui mentionne tous les termes expliqués en notes avec la page correspondante, un index des personnages (avec une brève information), un index des noms de personnes et de lieux, et un tableau de l'emboîtement des contes.

4 L'introduction de presque 140 pages est elle aussi au service du lecteur. Elle vise aussi à établir l'excellence de l'ouvrage de Galland. Elle comporte trois parties: l'une est biographique, la seconde littéraire, la troisième concerne la réception des Mille et une nuit. M. Couvreur n'apporte pas de documents nouveaux mais il utilise tous ceux qui sont bien connus ou ont été édités récemment (comme les journaux de Galland) et certaines correspondances ou témoignages, et leur examen très serré permet de restituer la chronologie la plus précise et donc les circonstances de temps, de lieu et de personnes, des événements de la vie de Galland, de son activité de savant et d'orientaliste, de traducteur et d'éditeur: chaque élément trouve ainsi son sens en particulier dans les situations et avec les protagonistes de chaque décision. La description des sources de Galland gagne ainsi en netteté, comme les motifs de la traduction des «Voyages de Sindbad » et la décision de les intégrer aux Mille et une nuit. On suit aussi très bien les raisons et les étapes des voyages de Galland en Orient comme de ses relations avec ses employeurs ou protecteurs. On découvre aussi dans le détail ses relations avec les « libraires » ses éditeurs, on comprend les motivations de Galland, les raisons de ses échecs et les compromis qu'il apprend à faire ou qu'il persiste à refuser. Une des informations les plus intéressantes concerne la dédicataire des Nuits, la marquise d'o fille de Guilleragues. M. Couvreur cherche en effet à montrer le rôle joué par les protectrices et les lectrices de Galland à qui il s'adresserait tout spécialement. La partie consacrée à la réception, qui fait une belle synthèse, insiste sur l'intérêt tout particulier des femmes pour le texte et en donne des témoignages choisis. Mais c'est que le texte de Galland met en valeur les femmes, leur volonté et leur culture : «Les Mille et une nuit peuvent être lues comme un manuel d'éducation à l'usage d'une future reine » (p. 129).

5 M. Couvreur présente avec tact les principes qui ont commandé la traduction de Galland et explique comment la suppression des poésies s'accompagne d'un travail de paraphrases et de gloses dans le texte même (l'ouvrage de Sylvette Larzul aurait pu être davantage sollicité). M. Couvreur examine avec précision aussi ce que le texte doit sinon à une oralité fondatrice, du moins à une représentation de l'oralité (M. Couvreur accrédite l'idée, combattue par Aboubakr Chraïbi, du statut oral de l'œuvre arabe). Il termine l'examen de la traduction en considérant le triple souci de la bienséance, des convenances et de la vraisemblance: tout ce qui fait des Nuits de Galland un texte " classique » (au sens que les XIX et Xx ${ }^{e}$ siècles donnent à ce mot). C'est aussi l'esprit qui inspire la partie littéraire de cette introduction intitulée "Un chef d'œuvre classique ». Il le serait en joignant "l'utile à l'agréable " (M. Couvreur traite avec une certaine réserve la question de la moralité de l'ouvrage), il le serait par l'articulation de la diversité (Les Mille et une nuit touchant selon M. Couvreur à l'épique et au tragique comme au comique et à l'ironique) à une unité globale qui serait même marquée par 
une belle symétrie (p. 125 : « le recueil jouissait d'une symétrie répondant aux prescrits d'un chef d'œuvre classique »). L'unité de l'ouvrage tiendrait d'abord à la maîtrise de Schéhérazade : M. Couvreur reprend l'idée que l'ensemble du texte dessine la ligne d'un traitement (à la fois psychique et moral) de la fureur de Schariar dont on peut reconstituer les étapes progressives jusqu'à la guérison finale. M. Couvreur veut également établir la qualité du livre sur sa composition : le manuscrit déjà respecterait les principes classiques et les choix faits au gré des circonstances par Galland auraient encore amélioré l'admirable organisation de l'ouvrage, dont M. Couvreur nous donne les éléments : tout prend place dans une harmonie complète. Selon lui,

En prolongeant les effets d'échos de séquences narratives entre les deux parties de l'œuvre, en poursuivant le fil psychologique qui préside à l'avancement du récit cadre, en assurant une circularité parfaite, Galland devait imaginer qu'il s'était simplement contenté de rendre sa perfection à une œuvre dont il admirait, dans son incomplétude, la magnifique cohérence : d'un chef d'œuvre multiforme de la littérature orale arabe, Galland avait tiré la matière de l'une des œuvres les plus formellement abouties de la littérature classique du règne de Louis XIV.

(p. 126: pour faire entrer le texte dans le lit de Procuste du classicisme, il faut cantonner le texte arabe dans la « littérature orale », contre l'évidence.)

On le voit, M. Couvreur s'attache d'un côté à la lettre (orthographe et ponctuation, lexique) du texte et de l'autre à sa composition : il voit l'ensemble, la structure, et il répond ainsi au défi de Muhsin Mahdi dans son édition de la partie des Nuits correspondant au principal manuscrit utilisé par Galland (donné à la bibliothèque du roi et qui porte son nom à la Bibliothèque nationale) : montrez que Galland a bien écrit une œuvre, a bien conçu le tout ! Oui, c'est le cas, dit M. Couvreur. Ce double regard sur la lettre et sur l'architecture conduit à laisser dans l'ombre des histoires ou les contes (l'usage associe les deux termes) : M. Couvreur ne les considère que dans la perspective de l'ensemble ou de la démonstration de la visée de Schéhérazade, et d'une certaine façon de la défense (et de la promotion) des femmes. C'est le seul élément du contenu qu'il envisage, car il contribue à l'unité parfaite du tout. On regrette qu'une édition savante et destinée à durer dans les bibliothèques ne fasse pas une présentation de chaque conte, de sa situation dans la mémoire du texte, de ses propriétés, de sa singularité, des rapports de Galland avec la tradition et des effets de ses décisions, de sa situation dans l'œuvre. Tout au plus M. Couvreur défend-il l'idée - qui suscite le débat - que les derniers contes sont ironiques.

On trouvera donc ici un texte sûr, dont les difficultés de détail sont levées (dans les notes), avec une présentation équilibrée qui rend l'auteur présent et donne du relief à son œuvre: on entre un peu dans son atelier, on entrevoit son idéal littéraire, on comprend mieux notre goût pour son ouvrage. Le passé des conditions de rédaction est restitué et l'œuvre doit parler pour elle-même, en même temps que l'admiration esthétique est sollicitée. M. Couvreur se rapproche ainsi de la tradition philologique française incarnée par Frédéric Deloffre. 


\section{AUTEURS}

JEAN-PAUL SERMAIN

Université Sorbonne Nouvelle - Paris 3, FIRL EA 174 